CARDIOVASCULAR MEDICINE

\title{
Paradox of circulating advanced glycation end product concentrations in patients with congestive heart failure and after heart transplantation
}

\author{
A Heidland, K Šebeková, A Frangiosa, L S De Santo, M Cirillo, F Rossi, M Cotrufo, A Perna, \\ A Klassen, R Schinzel, N G De Santo
}

Heart 2004;90:1269-1274. doi: 10.1136/hrt.2003.026989

See end of article for authors' affiliations

Correspondence to: Dr A Heidland, Department of Internal Medicine, University of Wurzburg, Hans-

Brandmann-Weg 1

D-97080 Wurzburg,

Germany; August.

Heidland@t-online.de

Accepted

25 February 2004

\begin{abstract}
Objectives: To analyse circulating concentrations of advanced glycation end products (AGEs) in patients with severe congestive heart failure (CHF) and after heart transplantation; to identify the potential contribution of kidney function to plasma AGE concentrations; and to determine whether AGE concentrations and parameters of oxidative stress are interrelated.

Methods and results: Circulating $N^{E}$-(carboxymethy)) lysine (CML) and AGE associated fluorescence (AGE$\mathrm{Fl}$ ), lipid peroxidation, and glomerular filtration rate (GFR) were measured in a cross sectional study of 22 patients with advanced CHF, 30 heart transplant recipients, and 20 healthy controls. Compared with the controls, the CHF patients had decreased CML (mean (SEM) $467.8(20.0) \mathrm{ng} / \mathrm{ml} v 369.3(22.3) \mathrm{ng} / \mathrm{ml}$, $p<0.01$ ), AGE-FI (mean (SEM) 302.2 (13.3) arbitrary units $v 204.9$ (15.7) arbitrary units, $p<0.01$ ), and GFR $(p<0.01)$. CML was positively related to decreased total protein and serum albumin and negatively to body mass index $(p<0.01)$. In contrast, in the heart transplant group, impaired GFR was associated with a notable rise of both CML (mean (SEM) $876.1(53.1) \mathrm{ng} / \mathrm{ml}, \mathrm{p}<0.01$ ) and AGE-Fl (mean (SEM) $385.6(26.1)$ arbitrary units, $p<0.01$ ). A positive relation between CML and serum albumin $(r=0.394, p<0.05)$ and lipofuscin $(r=0.651, p<0.01)$ was found.

Conclusions: The contrasting concentration of CML and AGE-Fl between patients with CHF and after heart transplantation in the presence of decreased GFR and oxidative stress are explained by lowered plasma proteins in CHF and higher concentrations in heart transplant recipients. In heart transplant recipients, in addition to myocardial inflammatory processes, immunosuppression may be important for enhanced formation of AGEs.
\end{abstract}

A dvanced glycation end products (AGEs) are a structurally diverse class of molecules, which are formed by a non-enzymatic reaction of reducing carbohydrates with primary amino groups of proteins and lipids. They accumulate slowly during aging but at an accelerated rate in diabetes, renal insufficiency, and hepatic failure. ${ }^{1-4}$ The plasma and tissue concentrations of AGEs are determined by hyperglycaemia (in diabetes mellitus), renal function, inflammation, and oxidative and carbonyl stress. ${ }^{5}$ Recently, food derived Maillard products were described as an important source of circulating AGEs. ${ }^{6}$ The toxicity of AGEs has been experimentally proved and arises from chemical modification and cross linking of proteins, as well as from cell activation through their receptors, including the AGE receptor. ${ }^{7}$ These interactions enhance free oxygen radical formation, with nuclear factor $\kappa \mathrm{B}$ activation and release of proinflammatory cytokines, adhesion molecules, and growth factors. ${ }^{89}$ Owing to these effects, AGEs are assumed to have an important pathogenetic role in the complications of diabetes, atherosclerosis, $\beta_{2}$ microglobulin amyloidosis, Alzheimer's disease, and cataract. ${ }^{10}$

Whereas circulating AGE concentrations and their potential toxicity were extensively studied in diabetes and renal insufficiency, ${ }^{11}{ }^{12}$ there is a lack of data in patients with congestive heart failure (CHF) and after heart transplantation. We hypothesised that their concentrations might be augmented, since under both conditions their renal removal may be impaired ${ }^{13}$ due to reduced renal function. Their endogenous formation may be enhanced due to oxidative stress. ${ }^{14}{ }^{15}$ Therefore, we analysed circulating AGE concentrations in patients with severe CHF and in patients after heart transplantation. Since most AGE products are poorly characterised, our interest was focused on $N^{\epsilon}$ (carboxymethyl)lysine (CML), which was shown to be a ligand of the AGE receptor. ${ }^{16}$ It originates not only from the classical pathway of AGE formation or auto-oxidation of glucose but also from lipid peroxidation and myeloperoxidase catalysed reactions. ${ }^{17}$ Moreover, we analysed plasma AGEassociated fluorescence (AGE-Fl). Our secondary aim was identifying the potential contribution of kidney function to plasma AGE concentrations. Finally, we sought to determine whether AGE concentrations and parameters of oxidative stress are interrelated.

\section{PATIENTS AND METHODS}

\section{Patient population and characteristics}

The study was carried out according to the Declaration of Helsinki and approved by the Institutional Ethics Board in Naples. Written informed consent was obtained from all participants.

\section{Congestive heart failure}

Twenty two patients (17 men, five women, age range 18-65 years) with advanced CHF (on the waiting list for heart

Abbreviations: AGE, advanced glycation end product; AOPP, advanced oxidation protein product; BMI, body mass index; CHF, congestive heart failure; $C M L, N^{E}$-(carboxymethyl)lysine; ELISA, enzyme linked immunosorbent assay; GFR, glomerular filtration rate; TNF $\alpha$, tumour necrosis factor $\alpha$ 
transplantation) were classified according to the New York Heart Association in functional classes III $(n=9)$ and IV $(n=13)$. Table 1 provides patients' characteristics. The main causes of CHF were ischaemic heart disease $(n=14)$, valvar heart disease $(\mathrm{n}=6)$, dilative cardiomyopathy $(\mathrm{n}=1)$, and aortic dissection $(\mathrm{n}=1)$. Four patients also had non-insulin dependent diabetes. All subjects had mild to moderate peripheral oedema and received standard medical treatment with loop diuretics (furosemide $25-500 \mathrm{mg} /$ day), angiotensin converting enzyme inhibitors (lisinopril 5-20 mg/day), aldosterone antagonists (spironolactone $100 \mathrm{mg} /$ day, $\mathrm{n}=8$ ), the $\beta$ blocker atenolol $(12.5-50 \mathrm{mg} /$ day, $\mathrm{n}=6)$, digoxin $(0.25-0.5 \mathrm{mg} /$ day $)$, aspirin, and oral nitrites in various combinations.

\section{Heart transplant recipients}

The population consisted of 30 cardiovascularly stable and oedema-free male patients (age range 18-60 years), who had received a transplant $1-12$ years previously, median follow up 5.33 years (table 1 gives clinical details). Eighteen heart transplant recipients were taking a triple immunosuppressive regimen (ciclosporin A $100-300 \mathrm{mg} / \mathrm{day}$, azathioprine $25-75 \mathrm{mg} /$ day, glucocorticosteroids $2.5-5 \mathrm{mg} /$ day) and 12 received only the combination of ciclosporin $\mathrm{A}$ and glucocorticosteroids. Normotension was achieved with diuretics (furosemide 25-100 mg/day, $\mathrm{n}=10$; hydrochlorothiazide $25 \mathrm{mg}, \mathrm{n}=7$ ) and the angiotensin converting enzyme inhibitor lisinopril (5-20 mg/day, $\mathrm{n}=22$ ). No patient was diabetic. Exclusion criteria were acute infections, malignancies, rheumatic disease, and primary wasting disorders.

\section{Healthy controls}

Twenty healthy age and sex matched participants (11 men, nine women, age range 25-60 years) served as controls (table 1).

\section{Methods}

\section{Blood sampling}

Venous blood was collected in the morning into $\mathrm{K}_{2}$-EDTA tubes after a fasting period of 10-12 hours and centrifuged for 10 minutes. The plasma was stored in aliquots at $-80^{\circ} \mathrm{C}$ till analysis.

Routine methods were used for blood chemical analysis of plasma creatinine, electrolytes, albumin, total protein, and liver enzymes.

\section{Fluorescence spectroscopy}

The fluorescence $\left(\lambda_{\mathrm{ex}} 370 \mathrm{~nm} / \lambda_{\mathrm{em}} 440 \mathrm{~nm}\right)$ of the 50 -fold diluted plasma samples was measured in triplicate on a FluoroMax spectrometer (Spex Instruments, Edison, New Jersey, USA) as described previously. ${ }^{18}$ Haemolytic samples were excluded. Although fluorescence is not very specific, the similar fluorescence spectra of various synthetic AGEs (including pentosidine and vesperlysines) and of serum supports the assumption that AGEs are the main fluorescent species in the serum. ${ }^{18}$ Moreover, the correlation of total fluorescence with AGEs, as measured by enzyme linked immunosorbent assay (ELISA) of imidazolone as well as competitive ELISA with the polyclonal antibody to unspecific AGEs, indicates that the chemical reactions leading to the generation of fluorescence and both antigenic epitopes are not identical but related. ${ }^{18}$ Albumin AGE content closely reflected total fluorescence.

\section{$N^{\epsilon}$-(carboxymethyl)lysine}

Plasma total CML concentrations were measured in triplicate after proteinase $\mathrm{K}$ digestion (to make hidden epitopes accessible) by a competitive ELISA developed by Roche Diagnostics (Penzberg, Germany) in a microtitre ELISA plate reader (Multiskan Ascent, Labsystem, Helsinki, Finland) at $405 \mathrm{~nm}$ with the CML monoclonal antibody 4G9 (Alteon Inc, Ramsey, New Jersey, USA) as described previously. ${ }^{11} \mathrm{~N}$ (carboxymethyl)-aminocaproic acid (Alteon Inc) served as a standard.

Advanced oxidation protein products (AOPPs) in plasma were determined according to Witko-Sarsat et al. ${ }^{19}$ Malondialdehyde was analysed by high performance liquid chromatography after derivatisation with thiobarbituric acid. Lipofuscin concentrations were determined turbidimetrically $^{20}$ and expressed in arbitrary units relative to the fluorescence of quinine sulphate on a standard curve.

Renal function was evaluated by the standard clearance technique of insulin clearance and $p$-aminohippuric acid clearance.

Body mass index (BMI) was determined by the ratio of body weight $(\mathrm{kg})$ to height $\left(\mathrm{m}^{2}\right)$.

\section{Statistical analysis}

Data are presented as mean (SEM). In the case of a normal distribution of the data, one way analysis of variance was used to test for significant differences between groups. If analysis of variance indicated that $\mathrm{p}<0.05$, Scheffe's post hoc test was applied. For all remaining parameters not

Table 1 Characteristics of healthy controls, patients with congestive heart failure (CHF) and patients after heart transplantation

\begin{tabular}{|c|c|c|c|}
\hline & Controls $(n=20)$ & CHF $(n=22)$ & Transplant $(n=30)$ \\
\hline Age (years) $\ddagger$ & $37.2(2.1)$ & $46.6(2.8)^{*}$ & $44.0(2.5)$ \\
\hline $\mathrm{SBP}(\mathrm{mm} \mathrm{Hg})$ & $126.0(2.1)$ & $109.3(3.4)^{* *}$ & $118.3(2.8)$ \\
\hline $\mathrm{DBP}(\mathrm{mm} \mathrm{Hg})$ & $76.5(1.7)$ & $73.9(2.9)$ & $76.5(1.6)$ \\
\hline BMI $\left(\mathrm{kg} / \mathrm{m}^{2}\right) \S$ & $24.5(0.7)$ & $26.6(0.4)^{* *}-$ & $26.9(0.5)^{* *}$ \\
\hline Sodium (mmol/l) & $138.4(0.6)$ & $135.5(1.5)$ & $137.3(0.5)$ \\
\hline Potassium (mmol//) & $4.09(0.06)$ & $4.24(0.11)$ & $4.19(0.10)$ \\
\hline Albumin (g/l) & $40.4(0.4)$ & $37.2(1.2)$ & $42.8(0.9) \dagger$ \\
\hline Total protein (g/l) & $69.1(0.9)$ & $56.9(1.1)^{\text {** }}$ & $70.9(0.6) \dagger$ \\
\hline Creatinine $(\mu \mathrm{mol} / \mathrm{l})$ & ND & 119.3 (8.7) & $138.5(7.2)$ \\
\hline Urea $(\mathrm{mmol} / /)$ & ND & $7.4(1.2)$ & $10.6(1.1)$ \\
\hline $\mathrm{C}_{\mathrm{ln}}\left(\mathrm{ml} / \mathrm{min} / 1.73 \mathrm{~m}^{2}\right)$ & $108.0(3.5)$ & $69.7(5.9)^{\star \star}$ & $66.4(4.8)^{\star \star}$ \\
\hline$C_{\text {PAH }}\left(\mathrm{ml} / \mathrm{min} / 1.73 \mathrm{~m}^{2}\right)$ & $439.4(10.5)$ & $331.6(18.9)^{* *}$ & $316.8(13.7)^{\star *}$ \\
\hline \multicolumn{4}{|c|}{$\begin{array}{l}\text { Data are mean (SEM). } \\
{ }^{*} \mathrm{p}<0.05 v \text { control group; }{ }^{* *} \mathrm{p}<0.01 v \text { control group; }+\mathrm{p}<0.01 v \mathrm{CHF} ; \ddagger \text { significance tested by analysis of variance } \\
\text { with Scheffe's post hoc test (parameters with normal distribution); } \text { ssignificance calculated after non-parametric } \\
\text { evaluation; } 9 \text { patients with peripheral oedema. } \\
\text { BMI, body mass index; } C_{\text {In }} \text {, insulin clearance; } C_{P A H}, p \text {-aminohippuric acid clearance; DBP, diastolic blood } \\
\text { pressure; ND, not determined; SBP, systolic blood pressure. }\end{array}$} \\
\hline
\end{tabular}


distributed normally, we used the Kolmogorov-Smirnoff test. In the case of significance the Mann-Whitney $U$ test with Bonferroni correction was used. Simple regression analysis for correlation studies was used. Significance was defined as $\mathrm{p}<0.05$.

\section{RESULTS \\ Patients with CHF}

Comparison with healthy controls

Patients in the CHF group were older than healthy controls and had lower systolic but not diastolic blood pressure (table 2). Their BMI was higher because of mild to moderate oedema. Plasma sodium concentration tended to be lower. Total protein concentration was significantly decreased and serum albumin was non-significantly lower. Renal function was significantly impaired: insulin clearance among the patients with CHF averaged $65 \%$ and $p$-aminohippuric acid clearance $75 \%$ of the control values. Plasma AGE-Fl (expressed directly or as ratio to plasma albumin), CML concentrations, and the CML to albumin ratio were significantly decreased (table 2). Parameters characterising lipid peroxidation-malondialdehyde and lipofuscin-were significantly increased but the AOPP concentrations were similar to those of the controls.

In the analysis of subgroups with ischaemic and valvar heart disease, no differences were observed concerning the AGE plasma concentrations. Similarly, in the four patients with non-insulin dependent diabetes mellitus the CML concentration and AGE-Fl corresponded to those of the whole group.

\section{Relation of AGE concentrations to renal function} When the concentrations of CML were compared with glomerular filtration rate (GFR), an inverse relation was observed $(r=0.434, \mathrm{p}<0.05)$, in contrast to AGE-Fl and the ratio of AGE-Fl to albumin.

\section{Relation of AGE concentrations to plasma sodium concentration}

A positive correlation between the concentration of plasma CML (but not AGE-Fl) and sodium $(r=0.457, \mathrm{p}<0.05)$ was established.

Correlation between plasma CML and BMI indicated an inverse relation (fig l).

\section{Comparison between AGEs and parameters of oxidative stress}

In the patients with $\mathrm{CHF}$, no relation between AGE and AOPP plasma concentrations was established. However,

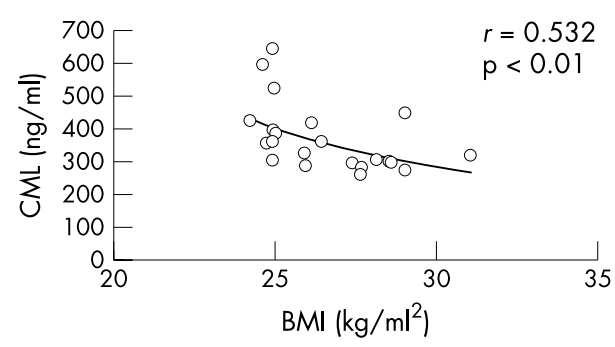

Figure 1 Relation between plasma $N^{E}$-(carboxymethyl)lysine (CML) concentration and body mass index (BMI) in 22 patients with congestive heart failure (CHF).

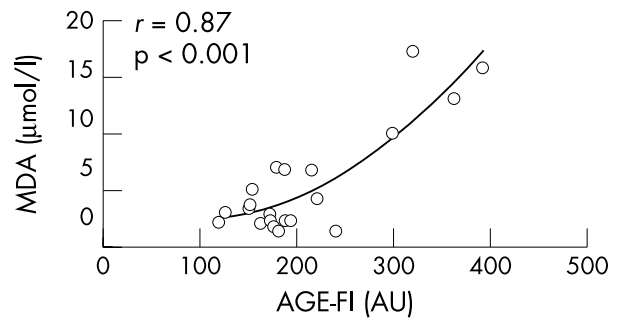

Figure 2 Relation between plasma malondialdehyde (MDA) and plasma advanced glycation end product associated fluorescence (AGEFl) in 22 patients with CHF. AU, arbitrary units.

fluorescent AGE concentrations correlated positively with malondialdehyde (fig 2) and with lipofuscin $(r=0.749$, $\mathrm{p}<0.001)$. Moreover, a positive relation between malondialdehyde and AOPPs was found $(r=0.697, \mathrm{p}<0.01)$.

\section{Relation of AGEs to plasma albumin}

A positive relation was shown for CML and albumin $(r=0.459, \mathrm{p}<0.01)$ but no relation between albumin and BMI was found. AGE-Fl and albumin were unrelated.

\section{Heart transplant recipients}

All heart transplant recipients were clinically stable with immunosuppressive treatment, without overt signs of transplant rejection. They were normotensive with antihypertensive treatment (table 2). Compared with the controls, the BMI of this group was increased: 20 were overweight (BMI $\left.25-30 \mathrm{~kg} / \mathrm{m}^{2}\right)$, three were obese $\left(\mathrm{BMI}>30 \mathrm{~kg} / \mathrm{m}^{2}\right)$, and only seven had a normal weight (BMI $21-25 \mathrm{~kg} / \mathrm{m}^{2}$ ). In comparison with the healthy controls and the CHF group, the heart transplant recipients had higher plasma concentrations of

Table 2 Plasma concentrations of AGEs, AOPPS, and parameters characterising lipid peroxidation in healthy controls and in patients with congestive heart failure (CHF) and after heart transplantation

\begin{tabular}{|c|c|c|c|}
\hline & Controls $(n=20)$ & $\mathrm{CHF}(n=22)$ & Transplant $(n=30)$ \\
\hline AGE-FI (AU) $\ddagger$ & 302.2 (13.3) & $204.9(15.7)^{* *}$ & $385.6(26.1)^{*}+\dagger$ \\
\hline AGE-FI:Alb (AU/g/l)‡ & $7.5(0.3)$ & $5.6(0.5)^{* *}$ & $9.1(0.7)^{*}+\dagger$ \\
\hline $\mathrm{CML}(\mathrm{ng} / \mathrm{ml}) \ddagger$ & $467.8(20.0)$ & $369.3(22.3)^{* *}$ & $876.1(53.1)^{\star *}+\dagger$ \\
\hline CML:Alb $(\mu \mathrm{g} / \mathrm{g}) \ddagger$ & $11.6(0.5)$ & $10.3(0.9)^{*}$ & $20.5(1.3)^{* \star}+\dagger$ \\
\hline AOPPs $(\mu \mathrm{mol} / \mathrm{l}) \S$ & $80.2(6.6)$ & $80.9(6.0)$ & $97.3(8.6)(n=15)$ \\
\hline MDA $(\mu \mathrm{mol} / \mathrm{I}) \ddagger$ & $1.9(0.3)$ & $5.4(1.0)^{* *}$ & $3.0(0.3)(n=17)$ \\
\hline Lipofuscin (AU)§ & $15.2(1.0)$ & $21.6(0.6)^{* *}$ & $22.6(1.3)^{* *}(n=18)$ \\
\hline
\end{tabular}

Data are mean (SEM).

${ }^{*} \mathrm{p}<0.05 v$ control group; ${ }^{* *} \mathrm{p}<0.01 v$ control group; $\mathrm{tp}<0.05 v \mathrm{CHF} ; \mathrm{\dagger}+\mathrm{p}<0.01 v \mathrm{CHF}$; $\ddagger$ significance calculated after non-parametric evaluation; §significance tested by analysis of variance with Scheffe's post hoc test (parameters with normal distribution).

AOPPs, advanced oxidation protein products; AGE-Fl, advanced glycation end product specific fluorescence; AGEs, advanced glycation end products; Alb, albumin; $A U$, arbitrary units; $C M L, N^{\epsilon}$-(carboxymethyl)lysine; MDA, malondialdehyde. 


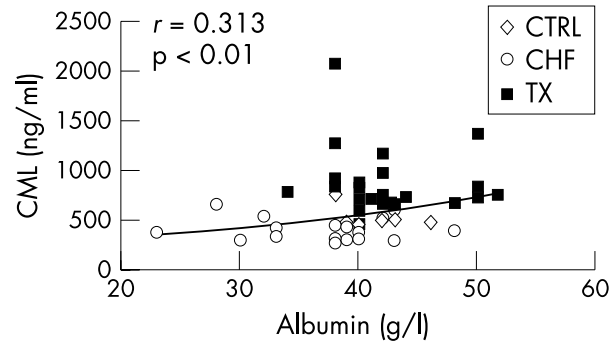

Figure 3 Relation between CML and albumin concentration in patients with CHF, heart transplant recipients (TX), and controls (CTRL).

total protein $(\mathrm{p}<0.01)$ and albumin $(\mathrm{p}<0.01)$. Renal function was significantly reduced: insulin clearance averaged $61.2 \%$ and the $p$-aminohippuric acid clearance $74 \%$ of the control values $(\mathrm{p}<0.01)$ (table 1). Plasma AGE-Fl $(32 \%)$ and the ratio of AGE-Fl to albumin $(20 \%)$ were significantly increased $(\mathrm{p}<0.01)$ (table 2$)$. Plasma CML concentrations were even higher (increased by a factor of 1.8 in comparison with the controls and 2.3 with the CHF groups, $\mathrm{p}<0.01$ ). When corrected for albumin, there was a rise by a factor of 1.7 and 1.9, respectively. The correlation between CML and albumin was significant $(r=0.394, \mathrm{p}<0.05)$. AOPP concentrations rose insignificantly (20\%) in comparison with the control and CHF groups. Malondialdehyde concentrations tended to be higher (44\%). Lipofuscin concentrations rose significantly compared with the control group $(\mathrm{p}<0.01)$. CML concentrations correlated positively with lipofuscin concentrations $(r=0.651, \mathrm{p}<0.01)$ but not with malondialdehyde concentrations. In a subgroup analysis of the patients taking triple immunosuppressive versus a double immunosuppressive treatment, no differences in the CML concentration and AGE-Fl was found.

\section{Correlation between AGE concentrations and plasma albumin of all groups}

When the participants of all the groups were evaluated together, both plasma AGE-Fl and plasma CML correlated positively with albumin (for CML, $r=0.313, \mathrm{p}<0.01$; and AGE-Fl, $r=0.475, \mathrm{p}<0.001)$, indicating that serum albumin has an important role in determining the concentration of plasma AGEs (figs 3 and 4).

\section{DISCUSSION}

\section{Congestive heart failure}

One major finding of the present study is that in patients with advanced CHF, regardless of the aetiology, the circulating AGE concentrations are not enhanced but slightly decreased. This observation does not, however, exclude the possibility of local AGE formation and accumulation in the cardiovascular system, in particular in atherosclerotic lesions. $^{210}$ The finding of lowered AGE concentrations in CHF is contrary to our expectations, since the patients presented the two main risk factors for AGE accumulation: impaired renal function and evidence of enhanced oxidative stress. In numerous experimental and clinical studies, the kidney was shown to have a key role in AGE removal. ${ }^{4}{ }^{11}$ Even in mild renal impairment, AGE concentrations may be higher than normal, reaching peak values in end stage renal failure. ${ }^{11}$ While the lowered AGE-Fl was unrelated to GFR, the association with CML was inverse. This shows that in CHF the negative relation between CML and GFR still exists, although at lower AGE concentrations.

Besides the role of impaired renal function in AGE accumulation, oxidative and carbonyl stress were shown to enhance AGE formation. ${ }^{5}$ In our patients with CHF, oxidative stress is suggested by the augmented concentrations of

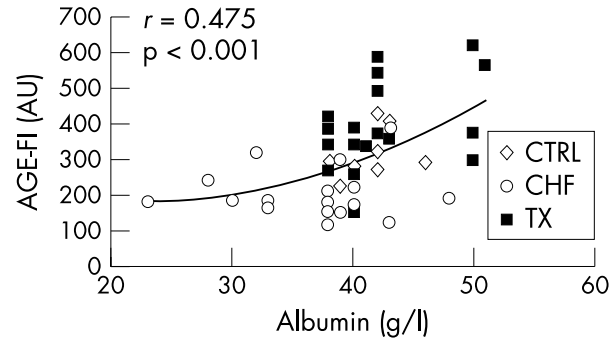

Figure 4 Relation between AGE-FI and albumin concentration in patients with $\mathrm{CHF}$, heart TX recipients, and CTRL.

malondialdehyde and lipofuscin-findings that are consistent with observations of other authors. ${ }^{21}$ However, plasma AOPP concentrations, which increase even with mild renal impairment in various kidney diseases, ${ }^{19}$ were similar to those of the healthy controls. AGE-Fl was closely related to lipid peroxidation products and, to a smaller degree, to CML, suggesting that in CHF, even at lower AGE concentrations, the causal link to oxidative stress is still evident.

Several mechanisms may account for the lower circulating AGE concentrations. Firstly, decreased circulating AGE concentrations in patients with CHF may be a consequence of dilution due to the hypervolaemic state, as indicated by clinical signs of congestion and peripheral oedema. This assumption is further supported by the positive relation between circulating CML and plasma sodium concentrations on the one hand and the negative relation to BMI on the other.

Secondly, since both AGE-Fl and CML are highly protein bound (more than $90 \%$ to albumin), ${ }^{18}{ }^{28-30}$ a parallel decline of these compounds is to be expected in the presence of a hypoproteinaemia. Accordingly, a positive correlation between CML and both total protein and albumin concentrations was found. Thus, the hypoproteinaemic state may directly contribute to the lower plasma CML concentration and AGE-Fl.

Thirdly, it has recently been shown that the dietary intake of AGEs contributes to the body AGE pool. ${ }^{6}$ Thus, food derived Maillard products are partially absorbed and may have a significant role in determining circulating AGE concentrations. ${ }^{622}$ In diabetic patients with normal renal function $^{6}$ and in non-diabetic patients with advanced renal failure, ${ }^{23}$ an AGE rich diet was followed by a rise of plasma AGE concentrations associated with an increase of proinflammatory cytokines. On the contrary, an AGE restricted diet greatly reduced the circulating AGE concentrations as well as their toxicity, also indicated by a lowering of proinflammatory cytokines. ${ }^{62}$ In patients with CHF, protein caloric malnutrition is common. Depending on the parameters measured, up to $50 \%$ are to some degree malnourished. ${ }^{24}$ The incidence of cardiac cachexia varies between $16 \%$ and $29 \% .^{25}$ In the development of wasting, besides anorexia, a catabolic-anabolic imbalance may be involved, ${ }^{27}$ probably through enhanced formation of tumour necrosis factor $\alpha(\mathrm{TNF}-\alpha) .{ }^{28}$ In our CHF group, plasma total protein was significantly reduced, possibly reflecting an impaired nutritional state. However, total protein and serum albumin concentrations are influenced by many other factors, such as fluid overload, inflammation, infection, or protein loss. In support of the assumption of a decreased protein caloric intake, other nutritional indicators were also altered. Compared with the heart transplant recipients, the patients with CHF had lower concentrations of blood urea $(-31 \%)$ and plasma creatinine $(-14 \%)$ in the presence of an identical decrease of GFR. A detailed analysis of the various nutritional parameters was beyond the scope of this study. 


\section{Heart transplantation group}

Another new finding of the present study is that in the heart transplantation group, the circulating AGE concentrations were significantly increased. This rise was even higher than would be expected from the decreased GFR when compared with earlier investigations in patients with diabetic nephropathy. ${ }^{11}$ In this regard, these observations correspond to our earlier findings of disproportionately increased AGE concentrations in paediatric kidney transplant recipients. ${ }^{3}$ With regard to the assumed dependency of circulating AGE concentrations on the plasma protein concentration, it is of note that both total protein and plasma albumin were significantly higher in the heart transplant recipients than in the CHF group and the healthy controls. Correspondingly, a direct, positive relation between CML and serum albumin was observed, similar to the studies in CHF. The dependency of AGEs on plasma albumin concentration is particularly striking when the patients with CHF, heart transplant recipients, and healthy controls were analysed together (figs 3 and 4).

Moreover, the transplanted heart may be a source of enhanced AGE formation through immune mediated inflammatory processes. Thus, an augmented myocardial expression of TNF- $\alpha$ and interferon $\gamma$ has been found after heart transplantation in relation to the development of transplant coronary artery disease. ${ }^{31}$ Considering, firstly, that inflammation leads to increased AGE formation ${ }^{32}$ and, secondly, that AGEs lead to increased radical formation and enhanced expression of proinflammatory cytokines, ${ }^{33}$ there is a possibility of a vicious cycle in which AGEs are the driving force, or at least a co-contributor. Lastly, it is assumed that immunosuppression with glucocorticoids and ciclosporin directly or indirectly contributes to enhanced AGE formation. Thus, glucocorticoids cause an overproduction of reactive oxidation species and thereby perturb nitric oxide availability, ${ }^{34}$ which is thought to lower AGE formation..$^{35}$ Moreover, glucocorticoids increase the appetite (and thereby the intake of food derived Maillard products), which probably contributes to the rise of BMI in the non-oedematous heart transplant recipients. Other authors reported a positive relation between prednisone treatment and increased concentrations of the AGE pentosidine after kidney transplantation. ${ }^{36}$ Similarly, ciclosporin may contribute to the heightened AGE accumulation, since it causes hypoxia and hydroxyl radical formation, while the production of nitric oxide is reduced. ${ }^{37}$ In line with the increased lipofuscin concentrations in our heart transplant recipients, ciclosporin was shown to enhance lipid peroxidation. $^{38}$

The impact of the increased circulating AGE concentrations on the prognosis of heart transplant recipients is unclear, but it is conceivable that this high burden, together with the numerous other risk factors, may contribute to the three long term complications of heart transplantation ${ }^{39}$ : development of cardiac allograft vasculopathy, progressive renal failure, and increased incidence of malignancies such as lymphoma and cell carcinomas of the skin. Thus, in in vitro investigations, AGEs were shown to exert genotoxic effects. ${ }^{40}$ Longitudinal follow up studies of circulating and tissue AGE concentrations, in particular in the coronary vessels, as well as administration of AGE inhibitors may address the question of what pathogenic role AGEs have in complications after heart transplantation.

It seems paradoxical that the plasma AGE concentrations in the patients with CHF were not increased despite the unfavourable prognosis of these patients (survival $<2$ years). However, in these patients we observed a lowering of plasma total protein concentration and thereby a decrease of an important binding site for both CML and AGE-Fl. Thus, when these AGEs are evaluated as a risk factor, serum proteins should always be taken into consideration. Additionally, the decline of plasma albumin per se was shown to have a detrimental effect on the prognosis of patients with CHF. In the majority of the published data, the risk of cardiovascular mortality is increased by a factor of two. ${ }^{41}$ The poor prognosis for CHF patients has been ascribed to a multitude of other risk factors, such as impaired renal function, upregulation of proinflammatory cytokines (interleukin 6 and TNF- $\alpha$ ), and augmented plasma concentrations of vasoactive peptides (angiotensin II, noradrenaline, endothelin I), particularly in patients with cardiac cachexia and oedematous decompensation. ${ }^{28}$

On the other hand, for the heart transplant recipients with high circulating AGE concentrations, the prognosis is much better than for patients with CHF. In Naples the 10 year survival rate averages $65-70 \%$. If the increased AGE concentrations are involved in long term complications of the heart transplant recipients, the use of compounds that lower AGE formation (renin-angiotensin system blockers, $\alpha$ lipoic acid, pyridoxal phosphate, or AGE cross link breakers) may contribute to an improved prognosis.

In summary, our data show that in CHF, circulating CML concentrations and AGE-Fl are decreased, in spite of impaired renal function and enhanced oxidative stress. Hypervolaemia, lowered plasma protein concentrations, and possibly decreased dietary AGE intake may be implicated. In contrast, after heart transplantation AGE concentrations are greatly augmented. This finding may be attributed to higher plasma protein concentrations. Moreover, potential myocardial inflammation processes and toxic effects of immunosuppression with glucocorticoids and ciclosporin may be involved. With regard to the pathogenic potential of AGEs in vitro and in vivo, they may have a role in long term complications after heart transplantation.

\section{Authors' affiliations}

A Heidland, A Klassen, Department of Internal Medicine, University of Wurzburg, Wurzburg, Germany

A Frangiosa, L S De Santo, M Cirillo, F Rossi, M Cotrufo, A Perna, N G De Santo, Chairs of Nephrology and Heart Surgery, Second University of Naples, Naples, Italy

K Šebeková, Institute of Preventive and Clinical Medicine, Slovak Medical University, Bratislava, Slovakia

R Schinzel, Institute of Physiological Chemistry, University of Wurzburg, Wurzburg, Germany

Funding: This study was supported by the Verein zur Bekämpfung der Hochdruck- und Nierenkrankheiten Würzburg e.V.

\section{REFERENCES}

1 Brownlee M. Advanced protein glycosylation in diabetes and aging. Annu Rev Med 1995;46:223-34.

2 Schleicher ED, Wagner E, Nerlich AG. Increased accumulation of the glycoxidation product $N$ (epsilon)-(carboxymethyl)lysine in human tissues in diabetes and aging. J Clin Invest 1997;9:457-68.

3 Šebeková K, Podracká L, Blažíček P, et al. Plasma levels of advanced glycation end products in children with renal disease. Pediatr Nephrol 2001;16:1105-12.

4 Šebeková K, Kupčová V, Schinzel R, et al. Markedly elevated levels of plasma advanced glycation end products in patients with liver cirrhosis: amelioration by liver transplantation. J Hepatol 2002;36:66-71.

5 Miyata T, Wada Y, Cai Z, et al. Implication of an increased oxidative stress in the formation of advanced glycation end products in patients with end-stage renal failure. Kidney Int 1997;51:1170-81.

6 Vlassara H, Cai W, Crandall J, et al. Inflammatory mediators are induced by dietary glycotoxins, a major risk factor for diabetic angiopathy. Proc Natl Acad Sci USA 2002;99:15596-601.

7 Schmidt AM, Yan SD, Wautier JL, et al. Activation of the receptor for advanced glycation end products: a mechanism for chronic vascular dysfunction in diabetic vasculopathy and atherosclerosis. Circ Res 1999;84:489-97.

8 Bierhaus A, Hofmann MA, Ziegler R, et al. AGEs and their interaction with AGE-receptors in vascular disease and diabetes. I. The AGE concept. Cardiovasc Res 1998;37:586-600. 
9 Thornalley PJ. Cell activation by glycated proteins, AGE receptors, receptor recognition factors and functional classification of AGEs. Cell Mol Biol 1998;44:1013-23.

10 Niwa T, Katsuzaki T, Miyazaki S, et al. Immunohistochemical detection of imidazolone, a novel advanced glycation end product, in kidneys and aortas of diabetic patients. J Clin Invest 1997;99:1272-80.

11 Wagner Z, Wittmann I, Mazak I, et al. $N^{E}$-(carboxymethyl)-lysine levels in type 2 diabetic patients: role of renal function. Am J Kidney Dis 2001;38:785-91.

12 Schinzel R, Münch G, Heidland A, et al. Advanced glycation end products in end-stage renal disease and their removal. Nephron 2001;87:295-303.

13 Herlitz H, Lindelöw B. Renal failure following cardiac transplantation. Nephrol Dial Transplant 2000;15:311-4.

14 Nakamura K, Kusano, K, Nakamura Y, et al. Carvedilol decreases elevated oxidative stress in human failing myocardium. Circulation 2002; 105:2867-71

15 Schimke I, Schikora M, Meyer R, et al. Oxidative stress in the human heart is associated with changes in the antioxidative defense as shown after heart transplantation. Mol Cell Biochem 2000;204:89-96.

16 Kislinger T, Fu C, Huber B, et al. $N^{\epsilon}$-(carboxymethyl)-lysine adducts of proteins are ligands for receptor for advanced glycation end products that activate cell signalling pathways and modulate gene expression. J Biol Chem 1999:274:31740-9

17 Fu M-X, Requena JR, Jenkins AJ, et al. The advanced glycation end product CML is a product of both lipid peroxidation and glycoxidation reactions. J Biol Chem 1996;271:9982-6.

18 Münch G, Keis R, Weßels A, et al. Determination of advanced glycation end products in serum by fluorescence spectroscopy and competitive ELISA. Eur J Clin Chem Clin Biochem 1997;35:669-77.

19 Witko-Sarsat V, Friedlander M, Khoa TN, et al. Advanced oxidation protein products as novel mediators of inflammation and monocyte activation in chronic renal failure. J Immunol 1998;161:2524-32.

20 Tsuchida M, Miura T, Mizunati K, et al. Fluorescent substances in mouse and human sera as a parameter of in vivo lipid peroxidation. Biochem Biophys Acta 1985;934:196-204.

21 Diaz-Velez DR, Garcia-Castineiras G, Mendoza-Ramos E, et al. Increased malondialdehyde in peripheral blood of patients with congestive heart failure. Am Heart J 1996;131:146-52.

22 Koschinsky T, He CJ, Mitsuhashi T, et al. Orally absorbed reactive glycation products (glycotoxins): an environmental risk factor in diabetic nephropathy. Proc Natl Acad Sci USA 1997;94:6474-9.

23 Uriabarri J, Peppa M, Cai W, et al. Restriction of dietary glycotoxins reduces excessive advanced glycation end products in renal failure patients. J Am Soc Nephrol 2003;14:728-31.

24 Carr JG, Stevenson LW, Walden JA, et al. Prevalence and hemodynamic correlates of malnutrition in severe congestive heart failure secondary to ischemic or idiopathic dilated cardiomyopathy. Am J Cardiol 1989;63:709-13.
25 Anker SD, Ponikowski P, Varney S, et al. Wasting as independent risk factor for mortality in chronic heart failure. Lancet 1997;349:1050-3.

26 Rauchhaus M, Doehner W, Francis DP, et al. Plasma cytokine parameters and mortality in patients with chronic heart failure. Circulation 2000;102:3060-7.

27 Poehlmann ET, Scheffers J, Gottlieb SS, et al. Increased resting metabolic rate in patients with congestive heart failure. Ann Intern Med 1994;121:860-2.

28 Anker SD, Chua TP, Ponikowski P, et al. Hormonal changes and catabolic/ anabolic imbalance in chronic heart failure and their importance for cardiac cachexia. Circulation 1997:96:526-34.

29 Miyata T, Ueda Y, Schinzato T, et al. Accumulation of albumin-linked and free-form pentosidine in the circulation of uraemic patients with end-stage renal failure: renal implications in the pathology of pentosidine. J Am Soc Nephrol 1996;7:1 198-206.

30 Gerdemann A, Wagner Z, Solf A, et al. Plasma levels of advanced glycation end products during haemodialysis, haemodiafiltration and haemofiltration: potential importance of dialysate quality. Nephrol Dial Transplant 2002; 17:1045-9

31 Ueland T, Sikkeland LI, Yndestad A, et al. Myocardial gene expression of inflammatory cytokines after heart transplantation in relation to the development of transplant coronary artery disease. Am J Cardiol 2003;92:715-7.

32 Anderson MM, Requena JR, Crowley JR, et al. The myeloperoxidase system of human phagocytes generates Nepsilon-(carboxymethyl)lysine on proteins: a mechanism for producing advanced glycation end products at sites of inflammation. J Clin Invest 1999;104:103-13.

33 Dukic-Stefanovic S, Gasic-Milenkovic J, Deuther-Conrad W, et al. Signal transduction pathways in mouse microglia $\mathrm{N}-11$ cells activated by advanced glycation endproducts (AGEs). J Neurochem 2003;87:2609-15.

34 luchi T, Akaike M, Mitsui T, et al. Glucocorticoid excess induces superoxide production in vascular endothelial cells and elicits vascular endothelial dysfunction. Circ Res 2003;92:81-7.

35 Asahi K, Ichimori K, Nakazawa H, et al. Nitric oxide inhibits the formation of advanced glycation end products. Kidney Int 2000;58:1780-7.

36 Hricik DE, Wu YC, Schulak A, et al. Disparate changes in plasma and tissue pentosidine levels after kidney and kidney-pancreas transplantation. Clin Transplant 1996;10:568-73.

37 Zhong Z, Arteel GE, Connor HD, et al. Cyclosporine A increases hypoxia and free radical production in the rat kidneys: prevention by dietary glycine. Am J Physiol 1998;275:F595-604.

38 Wang C, Salahudeen AK. Lipid peroxidation accompanies cyclosporine nephrotoxicity: effects of vitamin E. Kidney Int 1995;47:927-34.

39 Parameschwar J, Schofield P, Large S. Long-term complications of cardiac transplantation [editorial]. Br Heart J 1995;74:341-2.

40 Stopper H, Schinzel R, Sebekova K, et al. Genotoxicity of advanced glycation end products in mammalian cells. Cancer Lett 2003;190:151-6.

41 Dioussé L, Rothman KJ, Cupples LA, et al. Serum albumin and risk of myocardial infarction and all-cause mortality in the Framingham offspring study. Circulation 2002; 106:2919-24. 